\title{
Earman and Roberts on Empiricism about Laws*
}

\author{
Bradford Skow
}

\begin{abstract}
Earman and Roberts [2005] argue that a standard definition of "empiricism about laws of nature" is inadequate, and propose an alternative definition they think is better. But their argument against the standard definition fails, and their alternative is defective.
\end{abstract}

1. One standard definition of "empiricism about laws of nature" (or "humean supervenience about laws of nature") is:

$\left(E_{1}\right)$ The laws supervene on the occurrent, or non-nomic, facts. ${ }^{1}$

What does "non-nomic fact" mean? The term is hard to define, though there are paradigm cases: that all electrons have negative charge, for example.

Earman and Roberts [2005] argue that $\left(E_{1}\right)$ is a bad definition. In their argument they employ the following definition of "non-nomic fact":

$\left(D_{1}\right) F$ is a non-nomic fact iff $F$ "does not logically, or metaphysically, necessitate that anything is or is not a law of nature, or that any particular counterfactual conditional is true" (page 12).

For their argument against $\left(E_{1}\right)$ Earman and Roberts ask us to consider the following proposition:

*Published in Philosophy and Phenomenological Research 75 (2007): 158-162.

${ }^{1}$ [Earman 1986], [Carroll 1994]. 
(1) Some physical theory that achieves wide acceptance among human scientists in the 21 st century is true.

Earman and Roberts' argument proceeds as follows. It follows from $\left(D_{1}\right)$ that $(1)$ is a non-nomic fact: (1) does not necessitate that anything is or is not a law of nature, or that any particular counterfactual conditional is true. But if (1) is a non-nomic fact, then some alleged counterexamples to empiricism, as intuitively understood, are clearly not counterexamples to $\left(E_{1}\right)$. So $\left(E_{1}\right)$ is a bad definition of "empiricism about laws."

We don't need to rely on anything as complicated as (1) to generate this problem, though. Let $P$ be any lawlike sentence and consider the fact expressed by

(2) Either roses are blue or it is a law that $P$.

Suppose someone claims he has a counterexample to empiricism about laws: he describes two possible worlds, which apparently agree on the non-nomic facts but differ over whether it is a law that $P$. Suppose also that the two worlds agree that roses are red, not blue.

Now we argue as before: it follows from $\left(D_{1}\right)$ that $(2)$ is a non-nomic fact. Like (1), it does not necessitate that anything is or is not a law of nature, or that any particular counterfactual conditional is true. But the two worlds given do not agree on (2). So they do not agree on all non-nomic facts, and therefore do not constitute a counterexample to $\left(E_{1}\right)$. But even if the alleged counterexample to empiricism about laws is not genuine, it not does fail to be genuine for this reason. So $\left(E_{1}\right)$ is a bad definition of "empiricism about laws."

This argument turns on the fact that (2) is not a purely non-nomic fact. While (2) is a non-nomic fact according to definition $\left(D_{1}\right)$, it contains a nomic fact as a disjunct. So while it does not necessitate that anything is a law of nature, it in conjunction with another non-nomic fact - that roses are red (which obtains in each of the two possible worlds in question) — does necessitate that something is a law of nature. (1) works much the same way as (2): it behaves like an infinite disjunction of conjunctions ("Either $T_{1}$ achieves widespread acceptance and $T_{1}$ is true, or $T_{2}$ achieves widespread acceptance and $T_{2}$ is true...”) where each disjunct contain a 
nomic fact as one of its conjuncts. ( $T_{1}$, and the other theories, contain statements of the form "It is a law that $P . ")$

Neither my argument against $\left(E_{1}\right)$ using (2), nor Earman and Roberts' argument using (1), is a good one. The problem is with the arguments that (1) and (2) are non-nomic facts. Those arguments rely on $\left(D_{1}\right)$, but $\left(D_{1}\right)$ is a bad definition of "non-nomic fact." I've already noted that $\left(D_{1}\right)$ allows "mixed" facts, composite facts with nomic facts as components, to count as non-nomic facts. But there are other problems with $\left(D_{1}\right)$ too.

Certainly no empiricist who accepts $\left(E_{1}\right)$ will accept $\left(D_{1}\right)$; in fact it is part of their view that $\left(D_{1}\right)$ is false. Let $L$ be an actual law of nature and let $F$ be the conjunction of all the non-nomic facts about the actual world. Since empiricists think that the laws supervene on the non-nomic facts, empiricists think that $F$ metaphysically necessitates that $L$ is a law. So empiricists are committed to thinking that some non-nomic facts necessitate some nomic facts. But if empiricists accept $\left(D_{1}\right)$ they cannot say this.

It is not just empiricists who should reject $\left(D_{1}\right)$. Everyone else should as well. Everyone accepts that necessarily, if it is a law that $P$ then $P$ is true. So the fact that roses are red necessitates the fact that it is not a law that roses are not red. That is, the fact that roses are red necessitates that something is not a law of nature. If we accept $\left(D_{1}\right)$, then, it follows that the fact that roses are red is a nomic fact!

Earman and Roberts are aware that their argument turns on a bit of "logical trickery." They think that the way to formulate empiricism about laws to avoid this kind of trickery is to reject $\left(E_{1}\right)$, and not characterize the supervenience base for the laws as the set of non-nomic facts. I turn to their alternative proposal below. But what are the prospects for keeping $\left(E_{1}\right)$ and giving a better definition of "non-nomic fact"?

Earman and Roberts say they are following John Carroll in using $\left(D_{1}\right)$ as their definition of "non-nomic fact." But Carroll actually uses a different definition. His definition is

$\left(D_{2}\right) \quad F$ is a non-nomic fact iff $F$ can be expressed using only non-nomic concepts [Carroll 1994: page 58]. 
I do not myself endorse $\left(D_{2}\right)$. But it's worth noticing that none of the problems I raised for $\left(D_{1}\right)$ apply to $\left(D_{2}\right)$. It is compatible with $\left(D_{2}\right)$ that some non-nomic fact metaphysically necessitates some nomic fact. Similarly, according to $\left(D_{2}\right)$ a composite fact with a nomic fact as a component counts as a nomic fact. So (1) and (2) are nomic facts, on this definition. ${ }^{2}$

2. Earman and Roberts give an alternative formulation of empiricism about laws:

$\left(E_{2}\right)$ The laws supervene on the non-nomic facts that can be the output of a reliable, spatiotemporally finite observation or measurement procedure.

That this electron has negative charge and that the earth is eight light seconds from the sun appear to be in the supervenience base for the laws, according to $\left(E_{2}\right)$. But not (1): while there may be a procedure for measuring what scientists believe, there is no procedure for measuring whether they truly believe something.

$\operatorname{But}\left(E_{2}\right)$ is nevertheless a bad definition. Its defect is the opposite of the defect Earman and Roberts claim to find in $\left(E_{1}\right)$. There are pairs of possible worlds that are clearly not counterexamples to empiricism about laws, but are counterexamples to $\left(E_{2}\right)$.

Suppose $w_{1}$ and $w_{2}$ are governed by very different laws. Maybe the laws of $w_{1}$ are deterministic, while the laws of $w_{2}$ are probabilistic. Suppose also that $w_{1}$ and $w_{2}$ differ in their non-nomic facts. Maybe in $w_{1}$ particles always have definite positions, while in $w_{2}$ they do not. But suppose also that in $w_{1}$ it is, as a matter of law, impossible to measure the precise instantaneous state of a physical system. As a result of our ignorance in $w_{1}$, the subjective probability we assign, using the laws and what we do know, to the outcome of any measurement, is the same as the

\footnotetext{
${ }^{2}$ Things are a little tricky with (1), since it contains the truth predicate. Is the fact expressed by "The sentence 'It is a law that $P$ ' is true" a nomic fact, or not? You might argue that it is not; truth is a non-nomic concept, and the fact in question is just the fact that a certain sentence, a certain interpreted sequence of letters or sounds, falls under that concept.

The best way around this problem is to construe the truth-predicate as it appears in the above sentence and in (1) disquotationally. So " $S$ is true" expresses the same fact as $S$. If $S$ expresses a nomic fact, so does " $S$ is true."
} 
objective probability the laws in $w_{2}$ assign to the outcome of a similar measurement in $w_{2}$. So the laws of $w_{1}$ and of $w_{2}$ agree on which non-nomic facts can be the output of some reliable, spatiotemporally finite observation or measurement procedure; and (we may suppose) $w_{1}$ and $w_{2}$ agree on which of those special non-nomic facts obtain. Now, $w_{1}$ and $w_{2}$ are clearly compatible with empiricism about laws. But they are a counterexample to $\left(E_{2}\right)$. So $\left(E_{2}\right)$ is a bad definition of "empiricism about laws."

Earman and Roberts mention the possibility of "opaque" worlds, in which no non-nomic fact can be the output of a reliable, spatiotemporally finite observation or measurement procedure. They bite the bullet and deny that such worlds are possible. Maybe they would also deny that worlds like $w_{1}$, which aren't completely opaque, are possible. After all, they say, empiricists are already in the business of denying all sorts of modal intuitions.

But I think this case is different. The two possible worlds I described have a real-life source: $w_{1}$ and $w_{2}$ are incomplete descriptions of a world in which Bohm's version of quantum mechanics is true, and a world in which some collapse interpretation of quantum mechanics is true, respectively. Many take Bohm's version of quantum mechanics seriously, not merely as a theory that might be true in some possible world or other, but as a theory that might, for all we know, be true in our world. It would be wrong to rule it out a priori.

I conclude that Earman and Roberts have neither shown that $\left(E_{1}\right)$ is an inadequate definition of "empiricism about laws," nor have they proposed an adequate alternative definition in $\left(E_{2}\right)$. 


\section{References}

Carroll, John W. (1994). Laws of Nature. New York: Cambridge University Press.

Earman, John (1986). A Primer on Determinism. Boston: D. Reidel Publishing Company.

Earman, John and John T. Roberts (2005). "Contact with the Nomic: A Challenge for Deniers of Humean Supervenience about Laws of Nature, Part I: Humean Supervenience." Philosophy and Phenomenological Research 71(1): 1-22. 